\title{
OPTIMAL GROUP PREVENTIVE MAINTENANCE OF A SYSTEM WITH OBSERVABLE STATE PARAMETER
}

\author{
I. B. GERTSBAKH, ${ }^{*}$ Ben Gurion University of the Negev
}

\begin{abstract}
A group of $n$ independent identical machines with exponential lifetimes is considered. Repair is made simultaneously for all failed machines. It is possible to observe the 'state parameter', i.e. the number of operating machines. It is proved that for two types of critieria (minimal cost per time unit and maximal return per time unit) the optimal repair policy is of the following type: repair when the number of failed machines reaches some prescribed number $k, 0<$ $k \leqq n$.
\end{abstract}

GROUP MAINTENANCE: CRITICAL LEVEL: STOPPING TIME

We consider $n$ identical machines, the lifetime $T_{i}$ of each one being an exponential random variable: $T_{i} \sim \operatorname{Exp}(1)$. Machines fail independently; a unit cost is encountered for a unit of idle time of one machine. If at some instant $t^{*}$ a decision is made to repair, then all machines which are down at $t^{*}$ are renewed. It will be assumed that the renewal takes a negligible time, but the cost for renewing $k$ machines is $c_{0}+c_{1} . k, c_{0}, c_{1}$ being positive. At any time of system operation the information is available about the number of actually operating machines. Our goal is to find an optimal repair rule which minimizes the average long-run cost per unit of time.

Let $\left\{X_{t}, t \geqq 0\right\}$ be the random process describing the number of failed machines at time $t, X_{0}=0$. Denote by $\Gamma$ the class of all stopping times associated with $X_{t}$. We postulate that the stopping time $\tau_{n}=\inf \left\{t: X_{t}=n\right\} \in \Gamma$. Thus automatically for any $\tau \in \Gamma E[\tau] \leqq$ $E\left[\tau_{n}\right]<\infty$.

For a fixed $\tau$ the average long run cost per unit time equals the expected cost per cycle divided by the expected cycle length (see for example Ross (1983), p. 78):

$$
B(\tau)=\frac{E\left[c_{0}+c_{1} X_{\tau}+\int_{0}^{\tau} X_{\mathrm{s}} d s\right]}{E[\tau]} .
$$

In the following we assume that $0<c_{1}<1$. This is motivated by the natural demand that the cost paid for one machine's idle time during its average life $E\left[T_{i}\right]=1$ should exceed its repair cost $c_{1}$.

It will be shown that there is an optimal stopping rule $\tau^{*} \in \Gamma$ which minimizes $B(\tau)$ and that

$$
\begin{aligned}
\tau^{*} \in \Gamma^{*} & =\left\{\tau_{k}, k=1, \cdots, n\right\}, \\
\tau_{k} & =\inf \left\{t: X_{t}=k\right\} .
\end{aligned}
$$

\footnotetext{
Received 6 June 1984; revision received 6 September 1984.

* Postal address: Department of Mathematics and Computer Science, Ben Gurion University of the Negev, P.O.B. 653, Beer Sheva 84 105, Israel.
} 
In other words, the optimal replacement policy is to reset all failed machines at the moment when the number of failed machines reaches some prescribed number $k$. The search for the best $\tau_{k}$ in $\Gamma^{*}$ is an easy task. Indeed, one can see that

$$
B\left(\tau_{k}\right)=\frac{c_{0}+c_{1} \cdot k+\sum_{i=0}^{k-1}\left(m_{i+1}-m_{i}\right) \cdot i}{m_{k}}
$$

where

$$
\begin{aligned}
& m_{i}=E\left[\tau_{(i)}\right]=\sum_{q=0}^{i-1} 1 /(n-q), \quad j=1, \cdots, k, \\
& m_{0}=0 .
\end{aligned}
$$

A simple numerical check with (3), (4) will reveal the best $k$.

The fact that the optimal stopping rule $\tau^{*} \in \Gamma^{*}$ follows from a more general result due to Aven (1982) concerning the minimization problem of ratio-type functionals $B(\tau)=$ $M(\tau) / C(\tau)$ in the class $\tau \in \Gamma$.

$E\left[X_{t}\right]$ can be represented in an integral form

$$
E\left[X_{\tau}\right]=E\left[\int_{0}^{\tau} \alpha\left(X_{\mathrm{s}}\right) d s\right],
$$

where $\alpha(x)$ is the infinitesimal operator of $X_{t}$ (see Ross (1971), Formula (2) and Lemma 2.1(b)). In our case $\alpha(x)=n-x$. Denote by $\lambda^{*}=\inf _{\tau \in \Gamma} B(\tau)$. Obviously, $0<\lambda^{*}<\infty$.

One can prove, using the boundedness of $E[\tau]$ in $\Gamma$ that

$$
\inf _{\tau \in \Gamma} E\left[c_{0}+c_{1} X_{\tau}+\int_{0}^{\tau} X_{s} d s-\lambda^{*} \tau\right] \stackrel{\text { def }}{=} \inf _{\tau \in \Gamma} C\left(\lambda^{*}, \tau\right)=0
$$

(see Aven (1982), Theorem 2.1, and a similar statement in Ross (1971), Lemma 4.1). Replacing $E\left[X_{\tau}\right]$ according to (5) and $E[\tau]$ by $E\left[\int_{0}^{\tau} d s\right], C\left(\lambda^{*}, \tau\right)$ takes the following form:

$$
C\left(\lambda^{*}, \tau\right)=c_{0}+E\left[\int_{0}^{\tau}\left(\left(1-c_{1}\right) \cdot X_{s}+c_{1} \cdot n-\lambda^{*}\right) d s\right] .
$$

Proposition.

$$
\inf _{\tau \in \Gamma} C\left(\lambda^{*}, \tau\right)=\min _{\tau \in \Gamma^{*}} C\left(\lambda^{*}, \tau\right) .
$$

Proof. Note that $c_{1} \cdot n-\lambda^{*}<0$. If this were not true, $C\left(\lambda^{*}, \tau\right) \geqq c_{0}>0$, which contradicts (6). Since $X_{\mathrm{s}}$-process is non-decreasing, it follows immediately from (7) that the optimal stopping rule does exist and is defined as

$$
\tau^{*}=\inf \left\{t: X_{t} \geqq\left(\lambda^{*}-c_{1} n\right) /\left(1-c_{1}\right)\right\} .
$$

Clearly, $\tau^{*} \in \Gamma$ and this $\tau^{*}$ minimizes $B(\tau)$.

Okumoto and Elsayed (1983) considered the same group maintenance scheme, but they looked for the best age replacement policy. Obviously, the decision to repair all failed machines after a fixed time $t_{0}$ had elapsed since the last renewal is a particular choice of a stopping time and thus it cannot be superior to the best choice of $\tau$ in $\Gamma$. However, the difference in minimal costs is often rather small. For example, Okumoto and Elsayed had found that for $n=10$ machines, $c_{0}=\$ 10, c_{1}=\$ 2$, the optimal age for repair is $t_{0}=1 \cdot 2 \times$ (average machine's lifetime). It will result in an average cost of $\$ 8.17$ (the data given in their numerical example on page 673 are not correct due to an arithmetic error). Our computation shows that the optimal stopping rule is to reset the 
system immediately after $k=7$ machines had failed. This will give the average cost of $\$ 7 \cdot 99$.

It is worth noting that the search for optimal $k$ in (3) can be simplified by using the following interesting result of Aven (1982), Proposition 2.4: Let $B_{k}=B\left(\tau_{k}\right)$, where $\tau^{*}$ is the optimal stopping time corresponding to $k=k^{*}$. Then $B_{k}$ is non-increasing for $k \leqq k^{*}$, and $B_{k}$ is non-decreasing for $k \geqq k^{*}$.

The group preventive maintenance scheme considered above is the same as in the model of the so-called 'multiline system', described in Gertsbakh (1977), Chapter 3, Section 5. The difference lies in the cost functional. In the above book the problem was stated as the maximization of the expected return per unit of time, under the assumption that the repair of $k$ machines lasts time $d_{0}+d_{1} k, d_{0}>0, d_{1}>0$. The return functional is

$$
D(\tau)=\frac{E\left[\int_{0}^{\tau}\left(n-X_{s}\right) d s\right]}{E\left[\tau+d_{0}+d_{1} X_{\tau}\right]} .
$$

Let $\sup _{\tau \in \Gamma} D(\tau)=\mu^{*}$. A reasoning similar to that leading to (6) gives that the supremum over $\Gamma$ of the expression

$$
L\left(\mu^{*}, \tau\right)=E\left[\int_{0}^{\tau}\left(n-X_{\mathrm{s}}\right) d s-\mu^{*} \tau-\mu^{*} d_{0}-\mu^{*} d_{1} X_{\tau}\right]
$$

must be zero: $\sup _{\tau \in \Gamma} L\left(\mu^{*}, \tau\right)=0$.

Using (5) and $\alpha(x)=n-x$, we obtain that

$$
L\left(\mu^{*}, \tau\right)=-d_{0} \mu^{*}+E\left[\int_{0}^{\tau}\left(\left(1-d_{1}, \mu^{*}\right)\left(n-X_{s}\right)-\mu^{*}\right) d s\right] .
$$

As in the proposition (see also Ross (1974), Theorem 4.2(ii)), it is easy to check that

$$
\sup _{\tau \in \Gamma} L\left(\mu^{*}, \tau\right)=\max _{\tau \in \Gamma^{*}} L\left(\mu^{*}, \tau\right) .
$$

Indeed, $\left(1-d_{1} \cdot \mu^{*}\right)>0$, because otherwise for any $\tau \in \Gamma, L\left(\mu^{*}, \tau\right) \leqq-d_{0} \mu^{*}$. It is seen from (10) that the optimal stopping time is

$$
\tau^{*}=\inf \left\{t:\left(1-d_{1} \mu^{*}\right)\left(n-X_{t}\right)-\mu^{*} \leqq 0\right\}=\inf \left\{t: X_{t} \geqq n-\mu^{*} /\left(1-\mu^{*} d_{1}\right)\right\} .
$$

If all machines were operating without idle time then the average return would be $n /\left(1+d_{0}+n d_{1}\right)<n /\left(1+n d_{1}\right)$. This implies that $n-\mu^{*} /\left(1-\mu^{*} d_{1}\right)>0$.

A numerical investigation of (9) in the class $\Gamma^{*}$ was given by Gertsbakh (1977) without proving, however, the optimality in this class.

\section{References}

Aven, T. (1982) Optimal Replacement Times-a General Set-up. Statistical Research Report, Inst. of Statistics, Univ. of Oslo.

GerTSBAKH, I. B. (1977) Models of Preventive Maintenance. North-Holland, Amsterdam.

OKumoto, K. AND ElSAYED E. A. (1983) An optimum group maintenance policy. Naval Res. Logist. Quartz. 30, 667-674.

Ross, S. M. (1971) Infinitesimal look-ahead stopping rules. Ann. Math. Statist. 42, 297-303.

Ross, S. M. (1983) Stochastic Processes. Wiley, New York. 